\title{
THE PRESERVATION OF ATRIODICITY BY SEMICONFLUENT MAPPINGS
}

\author{
JAMES F. DAVIS
}

\begin{abstract}
Grace and Vought have proven that the image of a chainable continuum under a semiconfluent mapping is atriodic. This motivates the question of whether the semiconfluent image of an atriodic continuum is atriodic. In this paper this question is answered affirmatively.
\end{abstract}

In [1] Grace and Vought prove that the semiconfluent image of an arc-like continuum is atriodic. Since arc-like continua are atriodic, the question of whether semiconfluent mappings preserve atriodic continua naturally arises (Problem 8.5 of [5]). The main result of this paper is an affirmative answer to this question. Grace and Vought give an example [1, Example 1, p. 157] of a weakly confluent mapping from an arc-like continuum onto a simple triod. This shows that the result of this paper cannot be extended to weakly confluent mappings.

Definitions and statement of principal result. All spaces considered in this paper are metric. A continuum is a compact connected space and a mapping is a continuous function. A mapping $f$ of a continuum $X$ onto a continuum $Y$ is confluent if for each subcontinuum $K$ of $Y$, each component of $f^{-1}(K)$ is mapped by $f$ onto $K$; if for each subcontinuum $K$ of $Y$ and each two components $C_{1}$ and $C_{2}$ of $f^{-1}(K)$ either $f\left(C_{1}\right) \subset f\left(C_{2}\right)$ or $f\left(C_{2}\right) \subset f\left(C_{1}\right)$, then $f$ is semiconfluent; if for each subcontinuum $K$ of $Y$ there is a component of $f^{-1}(K)$ which is mapped by $f$ onto $K$, then $f$ is weakly confluent. Clearly confluent mappings are semiconfluent. Maćkowiak has shown [4, Corollary 3.2, p. 254; 5, Theorem 3.8] that semiconfluent mappings are weakly confluent. A continuum is a triod provided that it contains a subcontinuum whose complement has at least three components. A continuum is atriodic if it does not contain a triod. If $C$ is a collection of sets, $C^{*}$ denotes the union of the members of $C$.

The following theorem is our principal result. The initial part of the proof follows very closely the proof given by Grace and Vought for [1, Theorem 1, p. 152].

THEOREM 1. If $f$ is a semiconfluent mapping from an atriodic continuum $X$ onto the continuum $Y$, then $Y$ is atriodic.

Proof. Suppose that $Y$ contains a triod $W=A \cup B \cup C$, where $A, B$, and $C$ are continua and $A \cap B=A \cap C=B \cap C=Q$ is a proper subcontinuum of each of $A, B$, and $C$. Let $a, b$, and $c$ be points in $A \backslash Q, B \backslash Q$, and $C \backslash Q$, respectively.

Received by the editors July 16, 1985 and, in revised form, May 16, 1986. Presented to the 1986 Spring Topology Conference at the University of Southwestern Louisiana on April 3, 1986.

1980 Mathematics Subject Classification (1985 Revision). Primary 54F20; Secondary 54C10.

Key words and phrases. Semiconfluent mappings, atriodic continua.

This research was partially supported by a University of Richmond Faculty Summer Reasearch grant. 
If there is a component of $f^{-1}(A \cup B)$ which intersects $f^{-1}(a)$ but not $f^{-1}(b)$, then, by the semiconfluence of $f$, every component of $f^{-1}(A \cup B)$ that intersects $f^{-1}(b)$ also intersects $f^{-1}(a)$. Thus either every component of $f^{-1}(A \cup B)$ that intersects $f^{-1}(a)$ also intersects $f^{-1}(b)$ or every component that intersects $f^{-1}(b)$ also intersects $f^{-1}(a)$. Relabeling if necessary, we will suppose the former. Since $f$ is semiconfluent, it is weakly confluent. Let $X_{a c}$ be a component of $f^{-1}(A \cup C)$ such that $f\left(X_{a c}\right)=A \cup C$, and let $a^{\prime}$ and $c^{\prime}$ be points in $X_{a c}$ such that $f\left(a^{\prime}\right)=a$ and $f\left(c^{\prime}\right)=c$. Let $X_{a b}$ be the component of $f^{-1}(A \cup B)$ which contains $a^{\prime}$. Thus, $X_{a b}$ contains a point $b^{\prime}$ such that $f\left(b^{\prime}\right)=b$. Let $U_{a}, U_{b}$, and $U_{c}$ be open subsets of $Y$ containing $a, b$, and $c$ respectively such that $\bar{U}_{a}$ and $B \cup C \cup \bar{U}_{b} \cup \bar{U}_{c}, \bar{U}_{b}$ and $A \cup C \cup \bar{U}_{a} \cup \bar{U}_{c}$, and $\bar{U}_{c}$ and $A \cup B \cup \bar{U}_{a} \cup \bar{U}_{b}$ are mutually exclusive. Let $A_{0}, B_{0}$, and $C_{0}$ be the components of $A \backslash U_{a}, B \backslash U_{b}$, and $C \backslash U_{c}$, respectively, containing $Q$, and let $W_{0}=A_{0} \cup B_{0} \cup C_{0}$. Let $V_{a}=f^{-1}\left(U_{a}\right), V_{b}=f^{-1}\left(U_{b}\right)$, and $V_{c}=f^{-1}\left(U_{c}\right)$. Let

$$
B=\left\{J: J \text { is a component of } f^{-1}\left(W_{0}\right), J \cap \bar{V}_{a} \neq \varnothing, \text { and } J \cap \bar{V}_{b} \neq \varnothing\right\},
$$

and

$$
C=\left\{J: J \text { is a component of } f^{-1}\left(W_{0}\right), J \cap \bar{V}_{a} \neq \varnothing \text {, and } J \cap \bar{V}_{c} \neq \varnothing\right\} .
$$

Since $X_{a b}$ is a continuum intersecting both $f^{-1}(a) \subset V_{a}$ and $f^{-1}(b) \subset V_{b}, B$ is nonempty, and since $X_{a c}$ is a continuum intersecting both $f^{-1}(a) \subset V_{a}$ and $f^{-1}(c) \subset V_{c}, C$ is nonempty. Suppose that there is a member $J$ of $B$ such that $f(J)$ and $\bar{U}_{c}$ are mutually exclusive. From the semiconfluence of $f$ it follows that, if $L$ is a member of $C$, then $f(L)$ intersects $\bar{U}_{b}$. Thus either

(1) if $J$ is in $C, f(J)$ intersects $\bar{U}_{b}$, or

(2) if $J$ is in $B, f(J)$ intersects $\bar{U}_{c}$.

We will suppose (1). The argument in case (2) holds and is similar. Let

$$
\begin{aligned}
C_{0}=\{N: & N \text { is a component of } f^{-1}\left(A_{0} \cup C_{0}\right), \\
& \left.N \cap \bar{V}_{a} \neq \varnothing, N \cap \bar{V}_{c} \neq \varnothing, \text { and } N \subset X_{a c}\right\} .
\end{aligned}
$$

The remainder of the proof falls into three cases depending on the cardinality of $C_{0}$. We will now give an overview of these cases, postponing the details until after some helpful results have been proven. In each case we will reach a contradiction by constructing a triod in $X$. If $C_{0}$ has just one member, then a triod will be constructed with core $X_{a c}$ and arms extend toward $f^{-1}(a), f^{-1}(b)$, and $f^{-1}(c)$. If $C_{0}$ has exactly two members, a triod will be constructed with core including $X_{a c}$, with an arm extending toward $f^{-1}(b)$, and either two arms extending toward $f^{-1}(a)$ or two arms extending toward $f^{-1}(b)$. If $C_{0}$ has at least three components, a triod will be constructed with $X_{a c}$ as its core and with three arms extended toward $f^{-1}(b)$.

Preliminaries for the three cases. The following lemmas and theorem will be used in the consideration of the three cases mentioned above. We state them in somewhat more generality than what we need. In particular, note that the mapping in Lemma 2 need not be semiconfluent and that the spaces mentioned in Lemma 2 and Theorem 2 need not be continua.

Through the end of Lemma 2 we adopt the following hypothesis: Suppose that $A$ and $C$ are subcontinua of the continuum $M, M=A \cup C$, and $Q=A \cap C$ is 
connected. Suppose that $a$ is in $A \backslash C, c$ is in $C \backslash A$, and that $U_{c}$ is an open subset of $M$ which contains $c$ and whose closure does not intersect $A$. Let $C_{0}$ be the component of $C \backslash U_{c}$ which includes $A \cap C$.

LEMMA 1. The component of $M \backslash U_{c}$ which contains a is $A \cup C_{0}$.

ProOF. Let $K$ denote the component of $M \backslash U_{c}$ which contains $a$. Since $A \cup C_{0}$ is a connected set which contains $a$, and $A \cup C_{0} \subset(A \cup C) \backslash U_{0}$, it follows that $A \cup C_{0} \subset K$. Now suppose that $x$ is in $K$. If $x$ is in $A$ then $x$ is in $A \cup C_{0}$, so suppose that $x$ is not in $A$. Let $C_{1}$ be the closure of the component of $K \backslash A$ which contains $x$. By [6, Theorem 50, p. 18] $C_{1}$ intersects $A$. Since $C_{1} \cap A \subset A \cap C \subset C_{0}$, it follows that $C_{1} \cup C_{0}$ is connected. Since $C_{0}$ is a component of $C \backslash U_{c}$ and $C_{1} \cup C_{0} \subset C \backslash U_{c}$, we have that $C_{1} \subset C_{0}$. Thus $x$ is in $C_{0}$. Hence $K \subset A \cup C_{0}$, and we have that $K=A \cup C_{0}$.

LEMMA 2. Suppose that $X$ is a space, $f$ is a mapping of $X$ onto $M, X_{a c}$ is a component of $X$, and that $a^{\prime}$ and $c^{\prime}$ are points in $X_{a c}$ such that $f\left(a^{\prime}\right)=a$ and $f\left(c^{\prime}\right)=c$. Let $V_{a}=f^{-1}\left(U_{a}\right)$ and $V_{c}=f^{-1}\left(U_{c}\right)$. Then the component of $f^{-1}\left(A \cup C_{0}\right)$ which contains $a^{\prime}$ is the component of $X_{a c} \backslash V_{c}$ which contains $a^{\prime}$. Moreover, if $X_{a c}$ is compact, this component includes a component of $X_{a c} \backslash\left(V_{a} \cup V_{c}\right)$ which intersects both $\bar{V}_{a}$ and $\bar{V}_{b}$.

PrOOF. Let $H_{1}$ denote the component of $f^{-1}\left(A \cup C_{0}\right)$ which contains $a^{\prime}$, and let $H_{2}$ denote the component of $X_{a c} \backslash V_{c}$ which contains $a^{\prime}$. Since $A \cup C_{0} \subset(A \cup C) \backslash U_{c}$ we have that $f^{-1}\left(A \cup C_{0}\right) \subset f^{-1}\left(M \backslash U_{c}\right)=X \backslash V_{c}$. Thus $H_{1} \subset X \backslash V_{c}$ and hence $H_{1} \subset X_{a c} \backslash V_{c}$. Therefore $H_{1} \subset H_{2}$. Since $H_{2} \subset X \backslash V_{c}$, it follows that $f\left(H_{2}\right) \subset$ $M \backslash U_{c}$. The component of $M \backslash U_{c}$ which contains $a$ is $A \cup C_{0}$, by Lemma 1 , and $f\left(H_{2}\right)$ is a connected subset of $M \backslash U_{c}$ which contains $a$. Thus $f\left(H_{2}\right) \subset A \cup C_{0}$. Therefore $H_{2} \subset f^{-1}\left(A \cup C_{0}\right)$. Since $H_{2}$ is connected, $H_{1} \subset H_{2}$, and $H_{1}$ is a component of $f^{-1}\left(A \cup C_{0}\right)$, we have that $H_{1}=H_{2}$, the first conclusion of the lemma. Now suppose that $X_{a c}$ is compact, that is, it is a continuum. Since $X_{a c}$ intersects $V_{c}$, there is a point $c^{\prime \prime}$ in $H_{1} \cap \bar{V}_{c}$ by [6, Theorem 52(a), p. 21]. Let $T$ be the component of $H \backslash V_{a}$ which contains $c^{\prime \prime}$. Again by [6, Theorem 52(a), p. 21], T intersects $\bar{V}_{a}$ and thus is a component of $X_{a c} \backslash\left(V_{a} \cup V_{c}\right)$ which intersects both $\bar{V}_{a}$ and $\bar{V}_{c}$.

The following separation theorem will be used in Case II of our proof of Theorem 1.

THEOREM 2. Suppose that $X$ is a compact space, $U$ and $V$ are nonempty open subsets of $X$ whose closures are mutually exclusive, and there are just two components, $T_{1}$ and $T_{2}$, of $X \backslash(U \cup V)$ which intersect both $\bar{U}$ and $\bar{V}$. If each of $X \backslash U$ and $X \backslash V$ is the union of two mutually separated sets, one containing $T_{1}$ and the other containing $T_{2}$, then $X$ is the union of two mutually separated sets, one containing $T_{1}$ and the other containing $T_{2}$.

ProOF. Let $H_{1}$ and $H_{2}$ be mutually exclusive closed subsets of $X$ such that $T_{1} \subset H_{1}, T_{2} \subset H_{2}$, and $X \backslash V=H_{1} \cup H_{2}$. Let $K_{1}$ and $K_{2}$ be mutually exclusive 
closed subsets of $X$ such that $T_{1} \subset K_{1}, T_{2} \subset K_{2}$, and $X \backslash U=K_{1} \cup K_{2}$. Let

$$
\begin{aligned}
& H_{1}=\left\{I: I \text { is a component of } H_{1} \text { and } I \cap \bar{U} \neq \varnothing\right\}, \\
& H_{2}=\left\{I: I \text { is a component of } H_{2} \text { and } I \cap \bar{U} \neq \varnothing\right\}, \\
& \mathcal{K}_{1}=\left\{I: I \text { is a component of } K_{1} \text { and } I \cap \bar{V} \neq \varnothing\right\}, \\
& \mathcal{K}_{2}=\left\{I: I \text { is a component of } K_{2} \text { and } I \cap \bar{V} \neq \varnothing\right\} .
\end{aligned}
$$

For $i=1,2$, let $H_{i}^{\prime}=H_{i}^{*}$ and $K_{i}^{\prime}=K_{i}^{*}$. These four sets are closed. Clearly $H_{i}^{\prime} \subset H_{i}$ and $K_{i}^{\prime} \subset K_{i}$. Recalling that $H_{1}$ and $H_{2}$ are mutually separated and that $K_{1}$ and $K_{2}$ are mutually separated, we see that $H_{1}^{\prime} \cap H_{2}^{\prime}=\varnothing$ and $K_{1}^{\prime} \cap K_{2}^{\prime}=\varnothing$. Since $T_{1}$ intersects $\bar{U}$ and $T_{1} \subset H_{1}$, it follows that $T_{1}$ is a subcontinuum of a continuum in $H_{1}$. Thus $T_{1} \subset H_{1}^{\prime}$. Consequently, $T_{1}$ and $H_{2}^{\prime}$ are mutually exclusive. This, together with a similar argument with respect to $K_{1}$, gives us that $T_{1}$ and $H_{2}^{\prime} \cup K_{2}^{\prime}$ are mutually exclusive. Similarly, $T_{2}$ and $H_{1}^{\prime} \cup K_{2}^{\prime}$ are mutually exclusive.

Suppose that $p$ is in $X \backslash(U \cup V)$. Let $N$ denote the component of $X \backslash(U \cup V)$ which contains $p$. Suppose that $p$ is in $H_{1}^{\prime} \cup H_{2}^{\prime}$. Let $I$ be the component of $X \backslash V$ which contains $p$. The component of $I \backslash U$ which contains $p$ is $N$. From the definition of $H_{1}^{\prime}$ and $H_{2}^{\prime}$, we know that $I$ intersects $\bar{U}$, so it follows from [6, Theorem 51, p. 18] that the component of $I \backslash \bar{U}$ which contains $p$ has a limit point in $\bar{U}$. Therefore $N$ intersects $\bar{U}$. Similarly, if $p$ is in $K_{1}^{\prime} \cup K_{2}^{\prime}$, then $N$ intersects $\bar{V}$.

Suppose that $H_{1}^{\prime} \cup K_{1}^{\prime}$ and $H_{2}^{\prime} \cup K_{2}^{\prime}$ are not mutually exclusive. Then either $H_{1}^{\prime}$ intersects $K_{2}^{\prime}$ or $H_{2}^{\prime}$ intersects $K_{1}^{\prime}$. Supposing the former, let $p$ be a point in $H_{1}^{\prime} \cap K_{2}^{\prime}$. Since $H_{1}^{\prime} \cap V=\varnothing$ and $K_{2}^{\prime} \cap U=\varnothing$, we have that $p$ is in $X \backslash(U \cup V)$. Then the component $N$ of $X \backslash(U \cup V)$ which contains $p$ intersects both $\bar{U}$ and $\bar{V}$. Consequently either $N=T_{1}$ or $N=T_{2}$. This is contradictory, since $T_{1} \cap K_{2}^{\prime}=\varnothing$ and $T_{2} \cap H_{1}^{\prime}=\varnothing$. Thus, $H_{1}^{\prime}$ and $K_{2}^{\prime}$ are mutually exclusive. The argument that $H_{2}^{\prime}$ and $K_{1}^{\prime}$ are mutually exclusive is similar.

Therefore $H_{1}^{\prime} \cup K_{1}^{\prime}$ and $H_{2}^{\prime} \cup K_{2}^{\prime}$ are mutually exclusive closed subsets of $X$ containing $T_{1}$ and $T_{2}$ respectively. We should note here that $X$ is not necessarily the union of $H_{1}^{\prime} \cup K_{1}^{\prime}$ and $H_{2}^{\prime} \cup K_{2}^{\prime}$, since $X \backslash(U \cup V)$ may have components which intersect neither $\bar{U}$ nor $\bar{V}$.

Suppose that $X$ is not the union of two mutually exclusive closed sets, one containing $H_{1}^{\prime} \cup K_{1}^{\prime}$, the other containing $H_{2}^{\prime} \cup K_{2}^{\prime}$. Then there is a continuum $I \subset X$ which is irreducible between $H_{1}^{\prime} \cup K_{1}^{\prime}$ and $H_{2}^{\prime} \cup K_{2}^{\prime}$. Let $p$ be a point in $I \backslash\left(H_{1}^{\prime} \cup K_{1}^{\prime} \cup H_{2}^{\prime} \cup K_{2}^{\prime}\right)$. Since $U \subset H_{1}^{\prime} \cup H_{2}^{\prime}$ and $V \subset K_{1}^{\prime} \cup K_{2}^{\prime}, p$ is in $X \backslash(U \cup V)$.

Suppose $I \subset X \backslash(U \cup V)$. Let $q$ be a point in $I \cap\left(H_{1}^{\prime} \cup K_{1}^{\prime}\right)$. Suppose $q$ is in $H_{1}^{\prime}$. Let $J$ be the component of $H_{1}$ which contains $q$. Since $J \cup I$ is a connected subset of $X \backslash V$ which contains a point of $H_{1}$, and $H_{1}$ and $H_{2}$ form a separation of $X \backslash V$, it follows that $J \cup I \subset H_{1}$. Since $q$ is in $H_{1}^{\prime}, J$ intersects $\bar{U}$, and we have that $J \cup I \subset H_{1}^{\prime}$. This puts $p$ in $H_{1}^{\prime}$, a contradiction. We reach a similar contradiction if we suppose that $q$ is in $K_{1}^{\prime}$. Therefore $I \subset X \backslash(U \cup V)$.

Suppose that $I$ intersects $U$. Let $J$ be the component of $I \backslash(U \cup V)$ which contains $p$. Then $J$ intersects $\bar{U} \cup \bar{V}$. Suppose that $J$ intersects $\bar{U}$. Since $X \backslash V=H_{1} \cup H_{2}$, either $J \subset H_{1}$ or $J \subset H_{2}$. If $J \subset H_{1}$ then $J \subset H_{1}^{\prime}$ so $p$ is in $H_{1}^{\prime}$, a contradiction. If $J \subset H_{2}$, then $J \subset H_{2}^{\prime}$, also a contradiction. If $J$ intersects $\bar{V}$, on the other hand, similar contradictions arise. 
Therefore $X$ is the union of two mutually separated sets, one containing $H_{1}^{\prime} \cup K_{1}^{\prime}$, the other containing $H_{2}^{\prime} \cup K_{2}^{\prime}$. Since $T_{1} \subset H_{1}^{\prime} \cup K_{1}^{\prime}$ and $T_{2} \subset H_{2}^{\prime} \cup K_{2}^{\prime}$ we have the conclusion of the theorem.

The three cases. We now return to the proof of Theorem 1. The notation is that established in the first part of the proof.

For $N$ in $C_{0}$ let $\lambda(N)$ be the component of $f^{-1}\left(W_{0}\right)$ which contains $N$. If $N$ is in $C_{0}$, it follows that $\lambda(N)$ is in $C$ and thus $\lambda(N)$ intersects $\bar{V}_{b}$.

Case I. Suppose that $C_{0}$ has only one member, $T$. Let $H$ be the component of $f^{-1}\left(A \cup C_{0}\right)$ which contains $a^{\prime}$ and let $K$ be the component of $f^{-1}\left(A_{0} \cup C\right)$ which contains $c^{\prime}$. By Lemma 2, $H$ includes a component of $X_{a c} \backslash\left(V_{a} \cup V_{c}\right)$ which intersects both $\bar{V}_{a}$ and $\bar{V}_{c}$. Since $T$ is the only such component, $T \subset H$. That $T \subset K$ follows by a similar argument.

Thus $H, K$, and $\lambda(T)$ are three continua with a point in common (any point of $T$ ), no one of which is contained in the union of the other two. Thus, by Sorgenfrey's Theorem [7, Theorem 1.8, p. 443], $\lambda(T) \cup H \cup K$ contains a triod. This contradiction completes Case I.

Case II. Suppose that $C_{0}$ has just two members, $T_{1}$ and $T_{2}$. From Theorem 2 it follows that either both $T_{1}$ and $T_{2}$ are included in the same component of $X_{a c} \backslash V_{a}$ or both $T_{1}$ and $T_{2}$ are included in the same component of $X_{a c} \backslash V_{c}$. We will suppose the former. A similar argument holds in case the latter holds. Let $H$ be the component of $f^{-1}\left(A \cup C_{0}\right)$ which contains $a^{\prime}$ and let $K$ be the component of $f^{-1}\left(A_{0} \cup C\right)$ which contains $c^{\prime}$. By Lemma 2,K includes a component of $X_{a c} \backslash\left(V_{a} \cup V_{c}\right)$ which intersects both $\bar{V}_{a}$ and $\bar{V}_{c}$. Thus either $T_{1} \subset K$ or $T_{2} \subset K$ and hence $T_{1} \subset K$ and $T_{2} \subset K$. Also by Lemma 2 either $T_{1} \subset H$ or $T_{2} \subset H$. Let $n$ be the integer in $\{1,2\}$ such that $T_{n} \subset H$. Now $\lambda\left(T_{n}\right)$ is a continuum which intersects $\bar{V}_{b}$. Thus $H, K$, and $\lambda\left(T_{n}\right)$ are three continua with a point in common, no one of which is contained in the union of the other two. By [7, Theorem 1.8, p. 443] $H \cup K \cup \lambda\left(T_{n}\right)$ contains a triod. This is a contradiction to the atriodicity of $X$.

Case III. Suppose that $C_{0}$ has three members, $T_{1}, T_{2}$, and $T_{3}$. By [6, Theorem 52 , p. 21], there exist open subsets, $O_{1}, O_{2}$, and $O_{3}$, of $X$, such that $\bar{O}_{i}$ and $\bar{O}_{j}$ are mutually exclusive for $i \neq j$, and such that, for $i=1,2,3, T_{i} \subset O_{i}, f^{-1}\left(A_{0} \cup C_{0}\right)$ and bdry $\left(O_{i}\right)$ are mutually exclusive, and $O_{i}$ and $f^{-1}\left(U_{b}\right)$ are mutually exclusive. For each $i$, let $L_{i}$ be the closure of the component of $\lambda\left(T_{i}\right) \cap O_{i}$ which includes $T_{i}$. There is a point $p_{i}$ of $L_{i}$ in the boundary of $O_{i}$ by [6, Theorem 50, p. 18]. By the choice of $O_{i}, p_{i}$ does not belong to $f^{-1}\left(A_{0} \cup C_{0}\right)$, and thus $p_{i}$ does not belong to $X_{a c}$. Since $\bar{O}_{i}$ and $\bar{O}_{j}$ are mutually exclusive for $i \neq j$, it follows that $p_{i}$ is not in $L_{j}$.

Let $K_{i}=L_{i} \cup X_{a c}$ for $i=1,2,3$. Then $K_{i}$ is a continuum and, if $i \neq j$, $K_{i} \cap K_{j}=X_{a c}$, a proper subcontinuum of both $K_{i}$ and $K_{j}$. Thus $K_{1} \cup K_{2} \cup K_{3}$ is a triod.

Applications and questions. Grispolakis and Tymchatyn have proven [2, Corollaries 4.5 and 4.6 , pp. 352-353] that semiconfluent mappings preserve onedimensional acyclic continua and tree-like continua. They have recently proven $[\mathbf{3}$, Corollary 3.4] that acyclic atriodic continua are one dimensional (the author thanks the referee for bringing this paper to his attention). When we combine these results 
with Theorem 1 we see that semiconfluent mappings preserve important subclasses of the class of atriodic continua.

COROLlARY 1. Semiconfluent mappings preserve atriodic acyclic continua.

COROLlARY 2. Semiconfluent mappings preserve atriodic tree-like continua.

We close with some questions which are motivated by Theorem 1 and its proof. Suppose that $X$ and $Y$ are continua and that $f$ is a semiconfluent mapping of $X$ onto $Y$. Suppose $W \subset Y$ is a triod. In the proof of Theorem 1 we construct a triod $T \subset X$ such that $f(T) \subset W$. Can a triod $T \subset X$ be constructed such that $f(T)=W$ ? One might also ask whether Theorem 1 can be generalized to $n$-ods. That is, if $L$ is an $n$-od in $Y$, is there an $n$-od $K \subset X$ such that $f(K) \subset L$ (or $f(K)=L)$ ?

\section{REFERENCES}

1. E. E. Grace and E. J. Vought, Semi-confluent and weakly confluent images of tree-like and atriodic continua, Fund. Math. 101 (1978), 151-158.

2. J. Grispolakis and E. D. Tymchatyn, Semi-confluent mappings and acyclicity, Houston J. Math. 4 (1978), 343-357.

3.

4. T. Maćkowiak, Semi-confluent mappings and their invariants, Fund. Math. 79 (1973), 251-264.

5. _ Continuous mappings on continua, Dissertationes Math. (Rozprawy Mat.), No. 158, PWN, Warsaw, 1979.

6. R. L. Moore, Foundations of point set theory, Amer. Math. Soc. Colloq. Publ, vol. 13, Amer. Math. Soc., Providence, R. I., 1962.

7. R. H. Sorgenfrey, Concerning triodic continua, Amer. J. Math. 66 (1944), 439-460.

DEPARTMENT OF MATHEMATICS AND COMPUTER SCIENCE, UNIVERSity OF RiChMOND, RICHMOND, VIRGINIA 23173 